\title{
Counter movement jump and sport specific frequency speed of kick test to discrimi- nate between elite and sub-elite kickboxers
}

\author{
Süleyman Ulupınar ${ }^{1, *}$, Serhat Özbay ${ }^{2}$, and Cebrail Gençoğlü ${ }^{2}$ \\ ${ }^{1}$ Ermenek District National Education Directorate, Ministry of Education, Karaman, Turkey; and ${ }^{2}$ Faculty of Sport Sci- \\ ences, Erzurum Technical University, Erzurum, Turkey
}

Copyright: (c) 2020 S. Ulupınar et al. This is an open access article licensed under the Creative Commons Attribution License (https://creativecommons.org/licenses/by/4.0/).

\begin{abstract}
Background: Although counter movement jump (CMJ) test has been used for years to measure neuromuscular power, it is unclear what is the sensitivity of $\mathrm{CMJ}$ in distinguishing between combat athletes. Objective: The aim of this study is to assess the ability of 10-second frequency speed of kick test (FSKT) and CMJ to distinguish between elite and subelite kickboxing athletes. Methods: Twenty-four kickboxers voluntarily participated in this study. The inclusion criteria for all participants were to compete in the national championships at least the last three years. Athletes reaching at least a quarter-final were considered as elite $(n=12$, age $21.3 \pm 1.8$ years, body height $176.0 \pm 0.6 \mathrm{~cm}$, body mass $70.91 \pm$ $9.1 \mathrm{~kg}$, training experience $7.2 \pm 1.8$ years) whereas athletes who could not reach the quarter-finals were considered as sub-elite ( $n=12$, age $20.0 \pm 1.6$ years, body height $177.0 \pm 4.5 \mathrm{~cm}$, body mass $69.05 \pm 5.24 \mathrm{~kg}$, training experience 6.21 \pm 0.62 years) in the national championship held in the last tournament. Participants performed 10-second FSKT and $\mathrm{CMJ}$ test twice. Independent $t$-test was used to determine whether there were differences between groups and effect size (Cohen's $d$ ) was calculated. Additionally, a discriminant function analysis was used to determine which test most accurately distinguished elite and sub-elite kickboxers. Results: Body height, body mass, body mass index, and training experience did not significantly differ between the groups ( $p>.05)$, while CMJ $(p=.001, d=1.49)$ and FSKT $(p<$ $.001, d=2.56$ ) were significantly higher in the elite group than the sub-elite group. According to discriminant function analyses, FSKT correctly classified participation of $91.7 \%$ athletes in the elite and sub-elite groups, while CMJ correctly classified $70.8 \%$ of athletes. Conclusions: This study suggested that FSKT can be used to determine successful and non-successful kickboxers since it is more effective at distinguishing the groups than CMJ test.
\end{abstract}

Keywords: combat sports, martial arts, performance testing

\section{Introduction}

Jumping is a combined human movement that requires complex motor coordination between upper- and lowerbody parts. The mechanical process of the lower limbs during a vertical jump has been considered a suited movement pattern for evaluating explosive characteristics of various populations (Bosco \& Komi, 1979; Bosco \& Viitasalo, 1982; Markovic, Dizdar, Jukic, \& Cardinale, 2004). Vertical jump tests have been used in sports requiring explosive strength for diverse goals, including evaluating the lower limb neuromuscular power (Liebermann \& Katz, 2003), identifying

\footnotetext{
* Address for correspondence: Süleyman Ulupınar, Ermenek District National Education Directorate, Ministry of Education, Fikret Ünlü Street 10, 70400 Ermenek, Karaman, Turkey. E-mail: slymnulpnr@gmail.com
}

talented athletes (Stoessel, Stone, Keith, Marple, \& Johnson, 1991), and assessing motor fatigue (Hamilton, 2009; Haynes, Bishop, Antrobus, \& Brazier, 2019). For example, these tests have been commonly used to assess peak power of lower limbs in volleyball and basketball players (Duncan, Lyons, \& Nevill, 2008; Gathercole, Stellingwerff, \& Sporer, 2015; Haynes et al., 2019) or to evaluate motor changes in lower limb power all along a season in professional rugby athletes (Argus, Gill, Keogh, Hopkins, \& Beaven, 2009).

Kickboxing is a sport used kicks and punches with speed, stability, and strength (Podrigalo, Volodchenko, Rovnaya, Podavalenko, \& Grynova, 2018). The main purpose of the kickboxers during the match is to make an attack on the opponent with proper kicks and punches to plus points and gain the superiority (Gartland, Malik, \& Lovell, 2001; Hölbling, Preuschl, Hassmann, \& Baca, 2017; Ouergui, Hammouda, et al., 2013; 
Silva, Del Vecchio, Picanço, Takito, \& Franchini, 2011). Combat sports athletes, therefore, are expected to have high levels of anaerobic capacity for all-out attacks during the fight, also need a high aerobic energy system to replenish high-energy phosphates to maintain the anaerobic power-requiring attacks (Özbay \& Ulupınar, 2020; Podrigalo, Volodchenko, Rovnaya, \& Stankiewicz, 2017). With various methods such as proper and planned training and regular nutrition, kickboxers are trying to increase their competition performance (Franchini, Cormack, \& Takito, 2019; Reale, Slater, $\&$ Burke, 2017). The success in these sports are determined by technical-tactical excellence and supported by physical, physiological, and psychological development (Franchini et al., 2019; Hölbling et al., 2017).

In order to follow the physical and physiological developments, it is crucial to make performance measurements with valid tests. Although the countermovement jump (CMJ) test is commonly used for evaluation of explosive strength in various athletic populations, it seems that it does not reflect skill important for movement patterns in martial arts (Santos, Loturco, $\&$ Franchini, 2018). Conversely, the frequency speed of kick test (FSKT) appears as a sport-specific speed test for athletes in kickboxing and taekwondo. FSKT has been used to measure the performance level of the taekwondo athletes in recent studies (Bridge, Santos, Chaabene, Pieter, \& Franchini, 2014; Santos \& Franchini, 2018; Santos, Herrera-Valenzuela, Ribeiro da Mota, \& Franchini, 2016; Santos et al., 2018). We hypothesized that FSKT can also be valid and suitable for kickboxers. Thus, the aim of this study is to assess the ability of 10-second FSKT and CMJ to distinguish between elite and sub-elite kickboxing athletes.

\section{Methods}

This study was conducted according to the Declaration of Helsinki. Besides, the study protocol was approved by the Ethics Committee of Erzurum Atatürk University, Institute of Winter Sports and Sport Sciences.

\section{Participants}

Twenty four male kickboxers, who competed in the senior category of Turkey national kickboxing championship at least the last 3 years, and had kickboxing training at least five years, voluntarily participated in this study. Athletes reaching at least a quarter-final were considered as elite $(n=12$, age $21.3 \pm 1.8$ years, body mass $70.9 \pm 9.1 \mathrm{~kg}$, body height $176.1 \pm 5.9 \mathrm{~cm}$, training experience $7.2 \pm 1.8$ years) whereas athletes who could not reach the quarter-finals were considered as sub-elite $(n=12$, age $20.0 \pm 1.6$ years, body mass
$69.1 \pm 5.2 \mathrm{~kg}$, body height $177.1 \pm 4.5 \mathrm{~cm}$, training experience: $6.2 \pm 0.6$ years) in the national championship held in the last tournament. In this study, the elite group was created first, and sub-elite participants were selected in accordance with the weight category of each athlete in the elite group. Thus, performance changes related to physical properties were aimed to be eliminated. Descriptive characteristics for the participants are shown in Table 1. Participants and their coaches were informed about the experimental protocol. In addition, written informed consent was obtained from participants.

\section{Procedures}

The data collection process was carried out during the participant's weekly training routine. Participants performed a familiarization session at the beginning of the study. Additionally, participants were given the opportunity to warm-up and perform the tests at submaximal intensity before the main measurements. The tests were carried out between 10:00 and 11:30 AM. Participants were not permitted to use any supplements during the study period, or perform any exhaustive activity 24 hours before the testing days. Participants were verbally encouraged to maximize their performance in the tests.

\section{Anthropometrical measurements}

Participants' body heights were measured by Stadiometer (Portable Stadiometer, Holtain, Crymych, United Kingdom) and body mass was measured by Tanita TBF 401 (Tanita, Tokyo, Japan).

\section{Frequency speed of kick test}

After the warm-up protocol, participants performed 10 -second FSKT with their dominant leg in two repetitions. The number of repetitions during the FSKT was determined using a high-speed (HD $60 \mathrm{fps}$ ) video camera (Sony PXW-FX9, Sony, Tokyo, Japan) and timerepetition synchronization was checked in slow motion mode. After the commencing sound signal, the participants tried to reach the maximum number of kicks. Total kick number in each test was determined and used to describe the performance (Santos \& Franchini, 2018; Santos et al., 2016). In order for a kick to be considered a valid score, it had to be hit with the appropriate kicking technique to the marked area on the training sandbag. Kicks that did not hit the target were considered invalid. A total of two attempts were recorded, and the higher score was used for further evaluation (Santos, Herrera-Valenzuela, \& Franchini, 2019).

\section{Counter movement jump}

CMJ was measured using iPhone $\mathrm{X}$ mobile phone (Apple, Cupertino, CA, USA; HD $60 \mathrm{fps}$ ) with the My 
Jump 2 mobile application (Carlos Balsalobre; https:// apps.apple.com/app/my-jump-2/id1148617550), the validity and reliability of which were previously tested by Haynes et al. (2019). Participants were instructed to keep their hands on the hips, keep their knees in full extension, and then flex the knees and jump vertically at the fastest possible speed. If the subjects separated their hands at any stage of the jump, or if they pulled their knees in the flight phase of the jump, it was considered an error and the test was repeated. The jumps of the athletes who did not comply with the jump instructions were regarded as invalid and they were asked to repeat the jump again. After the first jump, the athletes were given 3-5 minutes of rest, and they performed second jump when they felt fully ready A total of two attempts were recorded, and the higher jump score was recorded in centimeters as a valid score.

\section{Statistical analysis}

Statistical analysis was performed using IBM SPSS Statistics (Version 21; IBM, Armonk, NY, USA). Significant level was stated $\alpha=.05$. Data were analyzed using descriptive statistics, and the results are presented as mean and \pm standard deviation. The normality of data was verified using the Shapiro-Wilk test. Independent $t$-test was used to determine whether there were differences between the elite group and sub-elite group. Effect sizes for the independent $t$-test were calculated by Cohen's $d$ formula (Cohen, 2013) and were classified according to Hopkins (2000) as < 0.2: trivial, 0.200.59: small, 0.60-1.19: moderate, 1.20-1.99: large, 2.00-3.99: very large, and $\geq 4$ : nearly perfect. Additionally, a discriminant function analysis was used to determine which test most accurately distinguished successful and non-successful kickboxers. The collinearity of data was analyzed to identify correlations between CMJ and FSKT. Since these tests were highly correlated $(r>$ .70) with each other, two discriminant function analysis model were used separately. The structural coefficient was used to determine the variables that discriminate between elite and sub-elite. A structural coefficient above .30 was considered as relevant for the interpretation of the linear vectors (Özbay \& Ulupınar, 2020).

\section{Results}

According to the comparison of the anthropometric characteristics and performance scores, FSKT and CMJ performance values were significantly higher in the elite group than sub-elite group $(p<.001$ and $d=$ 2.56; $p=.001$ and $d=1.49$, respectively) while there was no statistical difference at the age, body mass, body height, body mass index and training experience between the groups (Table 1).

Table 2 demonstrated that $\mathrm{CMJ}$ test correctly classified the elite and sub-elite kickboxers by $70.8 \%$, whereas FSKT correctly classified the groups by $91.7 \%$.

Table 1

Comparison of age, anthropometric characteristics, training experience and performance scores

\begin{tabular}{lrrrrr}
\hline & \multicolumn{1}{c}{ Elite } & \multicolumn{1}{c}{ Sub-elite } & \multicolumn{1}{c}{$t$} & \multicolumn{1}{c}{ Cohen's $d$} \\
\hline Age (years) & $21.3 \pm 1.8$ & $20.0 \pm 1.5$ & 1.905 & .070 & 0.78 \\
Body mass $(\mathrm{kg})$ & $70.9 \pm 9.1$ & $69.0 \pm 5.2$ & 0.615 & .545 & 0.26 \\
Body height $(\mathrm{cm})$ & $176.4 \pm 6.7$ & $177.3 \pm 4.5$ & -0.390 & .700 & 0.16 \\
Body mass index $\left(\mathrm{kg} / \mathrm{m}^{2}\right)$ & $22.7 \pm 1.8$ & $21.9 \pm 1.1$ & 1.261 & .220 & 0.54 \\
Training experience $($ years) & $7.2 \pm 1.8$ & $6.2 \pm 0.6$ & 1.779 & .098 & 0.75 \\
Counter movement jump (cm) & $38.88 \pm 3.35$ & $33.85 \pm 3.41$ & 3.643 & .001 & 1.49 \\
Frequency speed of kick test (repetition) & $23.42 \pm 1.67$ & $20.08 \pm 0.79$ & 6.226 & $<.001$ & 2.56 \\
\hline
\end{tabular}

Table 2

The classification of groups according to the discriminant function analyses between the counter movement jump and frequency speed of kick test

\begin{tabular}{|c|c|c|c|c|c|}
\hline \multirow[b]{3}{*}{ Group } & \multirow[b]{3}{*}{$n$} & \multicolumn{4}{|c|}{ Predicted group membership } \\
\hline & & \multicolumn{2}{|c|}{ Counter movement jump } & \multicolumn{2}{|c|}{ Frequency speed of kick test } \\
\hline & & Elite & Sub-elite & Elite & Sub-elite \\
\hline Elite & 12 & $75.0 \%(9)$ & $25.0 \%(3)$ & $83.3 \%(10)$ & $16.7 \%(2)$ \\
\hline \multirow[t]{2}{*}{ Sub-elite } & 12 & $33.3 \%(4)$ & $66.7 \%(8)$ & $0 \%(0)$ & $100 \%(12)$ \\
\hline & & \multicolumn{2}{|c|}{$70.8 \%$ of participants were correctly classified. } & \multicolumn{2}{|c|}{$91.7 \%$ of participants were correctly classified. } \\
\hline
\end{tabular}




\section{Discussion}

In this study, we examined the effectiveness of the sportspecific FSKT test in distinguishing between elite and sub-elite kickboxers compared to the CMJ test, which is widely used worldwide to evaluate lower-limb neuromuscular power (Liebermann \& Katz, 2003). Comparison between groups showed that both FSKT and CMJ performance values were significantly higher in the elite group than the sub-elite group (very large effect size, large effect size, respectively). However, FSKT correctly classified the groups by $91.7 \%$ while CMJ test correctly classified the elite and sub-elite kickboxers by $70.8 \%$. This study is the first investigation using FSKT in kickboxing athletes, which originally was designed to evaluate the sport-specific performances of taekwondo.

Different CMJ performances were presented in another study conducted by Tabben et al. (2014), in which the physical and physiological characteristics of high-level sports athletes were evaluated. Their study consisted of elite 19 judo, 19 karate and 16 taekwondo athletes, and CMJ performances of them on the mean of were $47.1,50.0$, and $43.2 \mathrm{~cm}$, respectively. Compared to that study, both groups of our study CMJ performances seem lower. Although it seems difficult to explain precisely, this difference can be explained by the different levels of training experiences and sport disciplines of the athletes. However, it has been reported that kicks are used a lot during kickboxing (Ouergui et al., 2016), taekwondo (Casolino et al., 2012) and karate competitions (Chaabene et al., 2014; Chaabene, Hachana, Franchini, Mkaouer, \& Chamari, 2012), and it is thought that these kicks need to be thrown at a certain speed in order to be accurate and, therefore, lower extremity muscle strength must be high in order to be successful in such sports (Santos \& Franchini, 2018; Tabben et al., 2014). Although various results have been reported according to sports characteristics, training experience, or competition status, CMJ seems a common test used to evaluate the lower limbs' power in combat sports athletes in the literature (Casolino et al., 2012; Santos et al., 2018; Slimani \& Chéour, 2016).

Although the FSKT test is a much newer test compared to the CMJ test, it has become more preferred in recent studies related to combat sports because it is thought to measure sports-specific skills (Santos \& Franchini, 2016, 2018; Santos et al., 2019, 2016, 2018). In another novel study carried out by Santos et al. (2019), they set a classification scale of FSKT for taekwondo athletes ( $\geq 22$ : excellent, 21: good, 17-20: regular, 16: poor and $\leq 15$ : very poor). With respect to this classification scale, the mean of FSKT scores of the elite group was good (mean: 23.42), but in the sub-elite group, the mean of FSKT scores was regular (mean: 20.08). There was never a poor or very poor score of FSKT in both groups. The reason for that can be explained by the fact that both study groups of the present study have training experience of at least 5 years and they selected from those who participate in the national competitions regularly.

A study reported an average of around 50 kicks during a kickboxing match (Slimani, Chaabene, Miarka, \& Chamari, 2017). Another study indicated that around 75 high-intensity actions were observed in a kickboxing match (Ouergui et al, 2014). In addition, Ouergui, Hssin, Franchini, Gmada, and Bouhlel (2013) emphasized that it is important to perform a single kick quickly and to be able to repeat the consecutive kicks rapidly in order to be successful in kickboxing. These studies show that the ability to kick quickly is one of the major predictors for kickboxing. Therefore, it is clear that the tests used to measure athletic performance must need to reflect the mechanical skills observed during the actual match. Under current circumstances, FSKT is one of the best alternatives to provide the mentioned requirement, but it is a limitation that it does not measure a skill related to punching. In addition, the fact that this study only included male athletes and a small sample size are other limitations of this study.

\section{Conclusions}

Even though the CMJ test is commonly used by coaches and sports scientists worldwide and an effective test for measuring lower limbs strength, it might not reflect specificity some sport discipline such as kickboxing. This study showed that FSKT can distinguish successful kickboxers from unsuccessful more effectively from CMJ test $(91.7 \%$ versus $70.8 \%$ ). Hence, it is suggested that FSKT tests can be used as an effective method for performance measurement in sports such as kickboxing taekwondo, muaythai, and karate which include kicking technique.

\section{Acknowledgments}

The authors gratefully thankthe participants for their cooperation during the research. This study was presented at El Ruha 7th International Conference on Social Sciences (August 3-5, 2020, American University of Libya, Tripoli, Libya).

\section{Conflict of interests}

There were no conflicts of interest. 


\section{References}

Argus, C. K., Gill, N. D., Keogh, J. W., Hopkins, W. G., \& Beaven, C. M. (2009). Changes in strength, power, and steroid hormones during a professional rugby union competition. Journal of Strength and Conditioning Research, 23, 1583-1592.

Bosco, C., \& Komi, P. V. (1979). Potentiation of the mechanical behavior of the human skeletal muscle through prestretching. Acta Physiologica Scandinavica, 106, 467-472.

Bosco, C., \& Viitasalo, J. T. (1982). Potentiation of myoelectrical activity of human muscles in vertical jumps. Electromyography and Clinical Neurophysiology, 22, 549-562.

Bridge, C. A., Santos, J. F. S., Chaabene, H., Pieter, W., \& Franchini, E. (2014). Physical and physiological profiles of taekwondo athletes. Sports Medicine, 44, 713-733.

Casolino, E., Cortis, C., Lupo, C., Chiodo, S., Minganti, C., \& Capranica, L. (2012). Physiological versus psychological evaluation in taekwondo elite athletes. International Journal of Sports Physiology and Performance, 7, 322-331.

Chaabene, H., Franchini, E., Miarka, B., Selmi, M., Mkaouer, B., \& Chamari, K. (2014). Time-motion analysis, physiological and rate of perceived exertion responses to karate official combats: Is there a difference between winners and defeated karatekas. International Journal of Sports Physiology and Performance, 9, 302-308.

Chaabene, H., Hachana, Y., Franchini, E., Mkaouer, B., \& Chamari, K. (2012). Physical and physiological profile of elite karate athletes. Sports Medicine, 42, 829-843.

Cohen, J. (2013). Statistical power analysis for the behavioral sciences (rev. ed.). New York, NY: Academic Press.

Duncan, M. J., Lyons, M., \& Nevill, A. M. (2008). Evaluation of peak power prediction equations in male basketball players. Journal of Strength and Conditioning Research, 22, 1379-1381.

Franchini, E., Cormack, S., \& Takito, M. Y. (2019). Effects of high-intensity interval training on olympic combat sports athletes' performance and physiological adaptation: A systematic review. Journal of Strength and Conditioning Research, 33, 242-252.

Gartland, S., Malik, M., \& Lovell, M. (2001). Injury and injury rates in Muay Thai kick boxing. British Journal of Sports Medicine, 35, 308-313.

Gathercole, R. J., Stellingwerff, T., \& Sporer, B. C. (2015). Effect of acute fatigue and training adaptation on countermovement jump performance in elite snowboard cross athletes. Journal of Strength and Conditioning Research, 29, $37-46$.

Hamilton, D. (2009). Drop jumps as an indicator of neuromuscular fatigue and recovery in elite youth soccer athletes following tournament match play. Journal of Australian Strength and Conditioning, 17(4), 3-8.

Haynes, T., Bishop, C., Antrobus, M., \& Brazier, J. (2019). The validity and reliability of the My Jump 2 app for measuring the reactive strength index and drop jump performance. Journal of Sports Medicine and Physical Fitness, 59, 253-258.

Hölbling, D., Preuschl, E., Hassmann, M., \& Baca, A. (2017). Kinematic analysis of the double side kick in pointfighting, kickboxing. Journal of Sports Sciences, 35, 317-324.
Hopkins, W. G. (2000). A new view of statistics: A scale of magnitudes for effect statistics. Retrieved from http://www. sportsci.org/resource/stats/effectmag.html

Liebermann, D. G., \& Katz, L. (2003). On the assessment of lower-limb muscular power capability. Isokinetics and Exercise Science, 11, 87-94.

Markovic, G., Dizdar, D., Jukic, I., \& Cardinale, M. (2004). Reliability and factorial validity of squat and countermovement jump tests. Journal of Strength and Conditioning Research, 18, 551-555.

Ouergui, I., Davis, P., Houcine, N., Marzouki, H., Zaouali, M., Franchini, E., ... Bouhlel, E. (2016). Hormonal, physiological, and physical performance during simulated kickboxing combat: Differences between winners and losers. International Journal of Sports Physiology and Performance, 11, 425-431.

Ouergui, I., Hammouda, O., Chtourou, H., Zarrouk, N., Rebai, H., \& Chaouachi, A. (2013). Anaerobic upper and lower body power measurements and perception of fatigue during a kick boxing match. Journal of Sports Medicine and Physical Fitness, 53, 455-460.

Ouergui, I., Hssin, N., Franchini, E., Gmada, N., \& Bouhlel, E. (2013). Technical and tactical analysis of high level kickboxing matches. International Journal of Performance Analysis in Sport, 13, 294-309.

Ouergui, I., Hssin, N., Haddad, M., Franchini, E., Behm, D. G., Wong, D. P., ... Bouhlel, E. (2014). Time-motion analysis of elite male kickboxing competition. Journal of Strength and Conditioning Research, 28, 3537-3543.

Özbay, S., \& Ulupınar, S. (2020). Strength-power tests are more effective when performed after exhaustive exercise in discrimination between top-elite and elite wrestlers. Journal of Strength and Conditioning Research. Advance online publication. doi:10.1519/JSC.0000000000003456

Podrigalo, L. V., Volodchenko, A. A., Rovnaya, O. A., Podavalenko, O. V., \& Grynova, T. I. (2018). The prediction of success in kickboxing based on the analysis of morphofunctional, physiological, biomechanical and psychophysiological indicators. Physical Education of Students, 22, 51-56.

Podrigalo, L. V., Volodchenko, A. A., Rovnaya, O. A., \& Stankiewicz, B. (2017). Analysis of martial arts athletes' goniometric indicators. Physical Education of Students, 21, 182-188.

Reale, R., Slater, G., \& Burke, L. M. (2017). Individualised dietary strategies for Olympic combat sports: Acute weight loss, recovery and competition nutrition. European Journal of Sport Science, 17, 727-740.

Santos, J. F. S., \& Franchini, E. (2016). Is frequency speed of kick test responsive to training? A study with taekwondo athletes. Sport Sciences for Health, 12, 377-382.

Santos, J. F. S., \& Franchini, E. (2018). Frequency speed of kick test performance comparison between female taekwondo athletes of different competitive levels. Journal of Strength and Conditioning Research, 32, 2934-2938.

Santos, J. F. S., Herrera-Valenzuela, T., \& Franchini, E. (2019). Establishing frequency speed of kick test classificatory tables in male and female taekwondo athletes. Kinesiology, 51, 213-218.

Santos, J. F. S., Herrera-Valenzuela, T., Ribeiro da Mota, G., \& Franchini, E. (2016). Influence of half-squat intensity 
and volume on the subsequent countermovement jump and frequency speed of kick test performance in taekwondo athletes. Kinesiology, 48, 95-102.

Santos, J. F. S., Loturco, I., \& Franchini, E. (2018). Relationship between frequency speed of kick test performance, optimal load, and anthropometric variables in black-belt taekwondo athletes. Ido Movement for Culture. Journal of Martial Arts Anthropology, 18(1), 39-44.

Silva, J. J. R., Del Vecchio, F. B., Picanço, L. M., Takito, M. Y., \& Franchini, E. (2011). Time-motion analysis in MuayThai and kick-boxing amateur matches. Journal of Human Sport and Exercise, 6, 490-496.

Slimani, M., Chaabene, H., Miarka, B., \& Chamari, K. (2017). The activity profile of elite low-kick kickboxing competition. International Journal of Sports Physiology and Performance, 12, 182-189.

Slimani, M., \& Chéour, F. (2016). Effects of cognitive training strategies on muscular force and psychological skills in healthy striking combat sports practitioners. Sport Sciences for Health, 12, 141-149.

Stoessel, L., Stone, M. H., Keith, R., Marple, D., \& Johnson, R. (1991). Selected physiological, psychological and performance characteristics of national-caliber United States women weightlifters. Journal of Strength and Conditioning Research, 5, 87-95.

Tabben, M., Chaouachi, A., Mahfoudhi, M., Aloui, A., Habacha, H., Tourny, C., \& Franchini, E. (2014). Physical and physiological characteristics of high-level combat sport athletes. Journal of Combat Sports and Martial Arts, 5(1), 1-5. 